\title{
Spondylodiscitis due to Cutibacterium acnes following lumbosacral intradiscal biologic therapy: a case report
}

\author{
Nicholas R Beatty*,1,2,3 (iD, Cole Lutz ${ }^{1}$, Kwadwo Boachie-Adjei ${ }^{1}$, Teresita A Leynes ${ }^{1}$, \\ Christopher Lutz ${ }^{1,4,5}$ \& Gregory Lutz $1,4,5$ \\ ${ }^{1}$ Regenerative Sportscare Institute, New York, NY 10128, USA \\ ${ }^{2}$ Department of Rehabilitation and Human Performance, Icahn School of Medicine at Mount Sinai, New York, NY, USA \\ ${ }^{3}$ Mount Sinai Hospital, New York, NY, USA \\ ${ }^{4}$ Hospital for Special Surgery, New York, NY, USA \\ ${ }^{5}$ New York Presbyterian, New York, NY, USA \\ *Author for correspondence: Tel.: +1 646870 7997; beattyn@regensportscare.com
}

\begin{abstract}
A 40-year-old woman with a history of chronic low back pain underwent a fluoroscopically guided intradiscal platelet-rich plasma injection (PRP) at the L5-S1 level. She subsequently developed progressive low back pain, night sweats and decreased ability to ambulate. Laboratory work-up revealed elevated acute phase reactants and imaging revealed L5-S1 intervertebral disc and vertebral end-plate abnormalities highly suggestive of spondylodiscitis. Computed tomography-guided aspiration and biopsy cultures grew Cutibacterium acnes and the patient was subsequently treated with intravenous antibiotics without surgical management. To the best of our knowledge, this is the first published case of lumbar spondylodiscitis following an intradiscal PRP injection, and brings to the forefront several clinically relevant issues including the antimicrobial effects of PRP, the role of $C$. acnes in spine infections and the ideal treatment protocol for intradiscal biologics in order to minimize morbidity and optimize functional outcomes.
\end{abstract}

First draft submitted: 14 January 2019; Accepted for publication: 25 July 2019; Published online: 19 August 2019

Keywords: Cutibacterium acnes $\bullet$ degenerative disc disease $\bullet$ discitis $\bullet$ intradiscal biologic therapy $\bullet$ intradiscal platelet-rich plasma $\bullet$ orthobiologics $\bullet$ regenerative medicine $\bullet$ spine care $\bullet$ spondylodiscitis

Low back pain associated with degenerative disc disease is extremely common affecting $40-90 \%$ of the population depending on inclusion criteria $[1,2]$ and is the leading cause of disease burden as measured by disability-adjusted life year (DALY) in most countries [3]. Total costs in the USA are estimated at US $\$ 200$ billion a year of which two-thirds is due to lost wages and lower productivity [4]. The disease burden is expected to continue to rise as the population ages and therefore improved treatment approaches are needed. To-date, there are few options to successfully treat the degenerative intervertebral disc conservatively [5] and there are challenges with disc replacement [6] and surgical interventions [7].

Targeted intradiscal biologic therapies (IBT) are a nonsurgical treatment option and have been studied more closely over the last two decades [8-11]. The native regenerative potential of the intervertebral disc declines with aging [10], and therefore may be augmented by autologous treatments including platelet-rich plasma (PRP), bone marrow aspirate concentrate, lipoaspirate and other molecules acting as cellular therapy, scaffolding, immunomodulating or nutritional/physiological support [12,13]. Ideal intradiscal therapy would inhibit disc catabolism, stimulate repair of the disc and improve the biomechanical status of the vertebral motion segment. Intradiscal PRP has been shown to improve functional outcomes at 6 months through 2 years without complications or adverse effects reported to date [14-18]. The antimicrobial properties of PRP have been well-studied [19-23] and offer a potential additional benefit when used in spine, musculoskeletal and soft-tissue conditions. However, it has not been fully elucidated whether it is the plasma/platelet components themselves or the presence of leukocytes [24] that confer antimicrobial benefit and this may play a role in deciding on treatment protocols. 


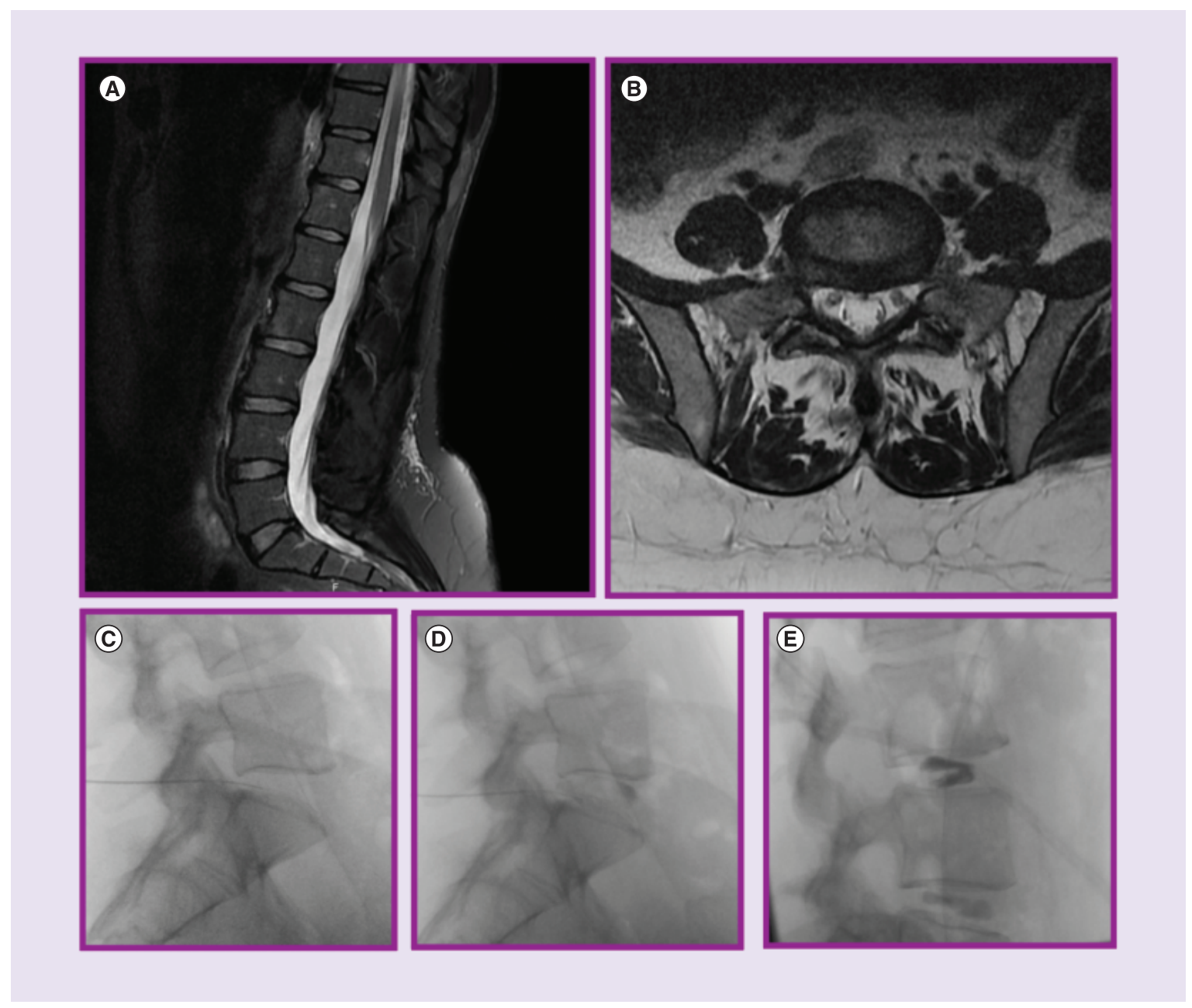

Figure 1. Preprocedure MRI and lumbar discography followed by L5-S1 intradiscal platelet-rich plasma.

Preprocedure Sagittal and Axial T2 MRI (A \& B). Lateral views highlighting the needle approach for L5-S1 (C \& D) and L4-L5 (E) discography.

\section{Case presentation}

A 40-year-old woman presented with severe chronic discogenic low back and buttock pain for 2 years not responding to comprehensive conservative treatment. As part of a diagnostic and therapeutic work-up and treatment plan, we performed fluoroscopic-guided lumbar discography at the L3-L4, L4-L5 and L5-S1 levels using a double-needle technique [25]. Sterile technique was practiced, including the use of sterile personal protective equipment, sterile drapes and equipment covers, and sterile skin applications (i.e., 10\% povidone-iodine solution followed by two applications of a sterile-package of $2 \% \mathrm{w} / \mathrm{v}$ chlorhexidine gluconate and $70 \% \mathrm{v} / \mathrm{v}$ isopropyl alcohol applicators, applied per product label specifications). At the L3-L4 and L4-L5 levels, there were normal nucleograms without evidence of annular disrupture and no concordant pain response. At the L5-S1 level, there was a grade III annular fissure [26] with $1 \mathrm{cc}$ of contrast and concordant symptoms. $60 \mathrm{cc}$ of peripheral blood was obtained from the right antecubital fossa and PRP was prepared using an Emcyte (FL, USA) protocol to provide $3 \mathrm{cc}$ of PRP. This protocol typically yields a high platelet concentration $(10-12 \times)$, low neutrophils and minimal to no red blood cells; no activation method was used.

She subsequently underwent an injection of $2.5 \mathrm{cc}$ PRP at the L5-S1 level with fluoroscopic guidance using a standard double-needle extrapedicular technique under monitored anesthesia care (Figure 1). A contrast agent (Omnipaque 180, GE Healthcare, MA, USA) was utilized without intradiscal antibiotics. Again, sterile technique was practiced throughout the entire procedure and handling of the PRP. The procedure was well-tolerated without any immediate complications. 
Several weeks following the injection her back pain started to increase which included night sweats and decreased mobility. Follow-up imaging was delayed due to international healthcare system triaging policies and a lumbar MRI was ultimately performed in the USA 10 weeks following the procedure with findings concerning for discitis (Figure 2). Laboratory studies revealed a normal CBC with differential, ESR $9 \mathrm{~mm} / \mathrm{h}$ and CRP $39.3 \mathrm{mg} / \mathrm{l}$. She was admitted to a tertiary care center for further work-up and treatment.

CT Scan of the lumbar spine with contrast revealed destruction of the end-plates bordering the L5-S1 IVD, more prominent in the superior endplate, extension of lytic bone marrow destruction to the inferior L5 marrow space, and reactive sclerosis in the marrow space of S1, with preservation of the L5-S1 disc height. MRI of the lumbar spine with and without contrast revealed L5-S1 cortical irregularity, erosions of adjacent endplates with patchy enhancement, and a T2 hyperintense lesion within the inferior L5 vertebral body endplate demonstrating peripheral enhancement corresponding to CT. There was no epidural or soft tissue abscess.

Due to ongoing concern for infection, L5-S1 disc aspiration and biopsy were carried out. The aspirate was bloody and purulent fluid, and the culture results grew a Gram-positive bacillus. Final culture grew a heavy growth of Cutibacterium acnes. After a discussion of the possibility of skin flora contamination and potential utility of repeating the biopsy, the patient declined. Given the heavy growth, purulent fluid, lack of other microbial culture and known but rare risk of $C$. acnes causing postsurgical infection, treatment was indicated and antibiotics were administered (i.e., ceftriaxone 2 gm iv. q $24 \mathrm{~h} \times 6$ weeks; a peripherally inserted central catheter was utilized).

After completion of antibiotic treatment, follow-up labs revealed: ESR $2 \mathrm{~mm} / \mathrm{h}, \mathrm{CRP} 4.1 \mathrm{mg} / \mathrm{l}$ and normal $\mathrm{CBC}$ with differential. Repeat CT scan revealed prominent destruction of the inferior endplate of $\mathrm{L} 5$ and to a lesser degree the superior endplate of S1 with well-demarcated and prominent area of surrounding sclerosis in keeping with the previously treated infectious process. There was no ongoing destruction of the bone observed to suggest an active infectious process. Clinically, she remained afebrile. Back pain persisted but was less severe and her functional status had improved from its nadir. Follow-up medical and surgical evaluation at 1-year postinjection concluded that there was no longer a sign of active infection, no spinal instability and no need for spinal fusion.

\section{Discussion}

Infectious complications following discography are uncommon, reported as $0.016 \%$ per examination and $0.0054 \%$ per disc level [27]. Most patients have monomicrobial infection with Staphylococcus aureus accounting for more than $50 \%$ of cases [28]. Other causes include nonpyogenic streptococci, including viridans group, milleri group, Streptococcus bovis and enterococci; pyogenic streptococci, including groups B and C/G; Pseudomonas aeruginosa, coagulase-negative staphylococci, Candida spp., tuberculous infection; and brucellosis. Skin contaminants and mixed flora have been cultured as well.

C. acnes (formerly Propionibacterium acnes) is an aerotolerant anaerobic Gram-positive rod-shaped bacterium that resides as normal flora on the human skin and in the oral cavity, conjunctiva, intestinal tract and external ear canal [29]. While $C$. acnes can be prevalent in intervertebral discs of asymptomatic patients [30], C. acnes is also a well-established pathogenic bacterium resulting in infections of bone and joints [31], surgical implants [32], vertebral osteomyelitis, pyogenic and nonpyogenic discitis [33-35], and spondylodiscitis [36]. In our case, C. acnes was isolated on biopsy with heavy growth and associated pus consistent with pyogenic spondylodiscitis. This species and its subtypes are an under-recognized pathogen in spine infections, complicated by its more indolent clinical presentation, minimal laboratory signs and challenges in culture due to questions of its role as a pathogen or contaminate.

Spondylodiscitis following the use of IBT is previously not reported and IBT has a better safety profile compared to other treatments such as oral corticosteroids, epidural steroid injections, and surgery. However, this case highlights the potential morbidity associated with IBT infections. IBT treatment requires vigilance in sterile technique, including biologic injectate preparation; risk-stratification of patients who might be at higher risk for infection; and protocol optimization, based on better understanding of the mechanisms involved in the pleiotropic effects of PRP and its interactions with the in vivo intervertebral disc environment. In the context of $C$. acnes - which can resist phagocytosis and persist in macrophages, mediate inflammatory cascades, and exhibit cell-aderant properties that may play a role in biofilm formation $[31,37]$ - the preparation, components, and antimicrobial activity of PRP need further study.

Primary technical and procedural considerations for IBT include the use of intradiscal biologics and optimization of the components of the PRP. Traditional provocative discography often includes the use of intradiscal, intravenous, or intradiscal plus intravenous antibiotics (e.g. cefazolin) [38,39]. Intradiscal antibiotics are often mixed with the 

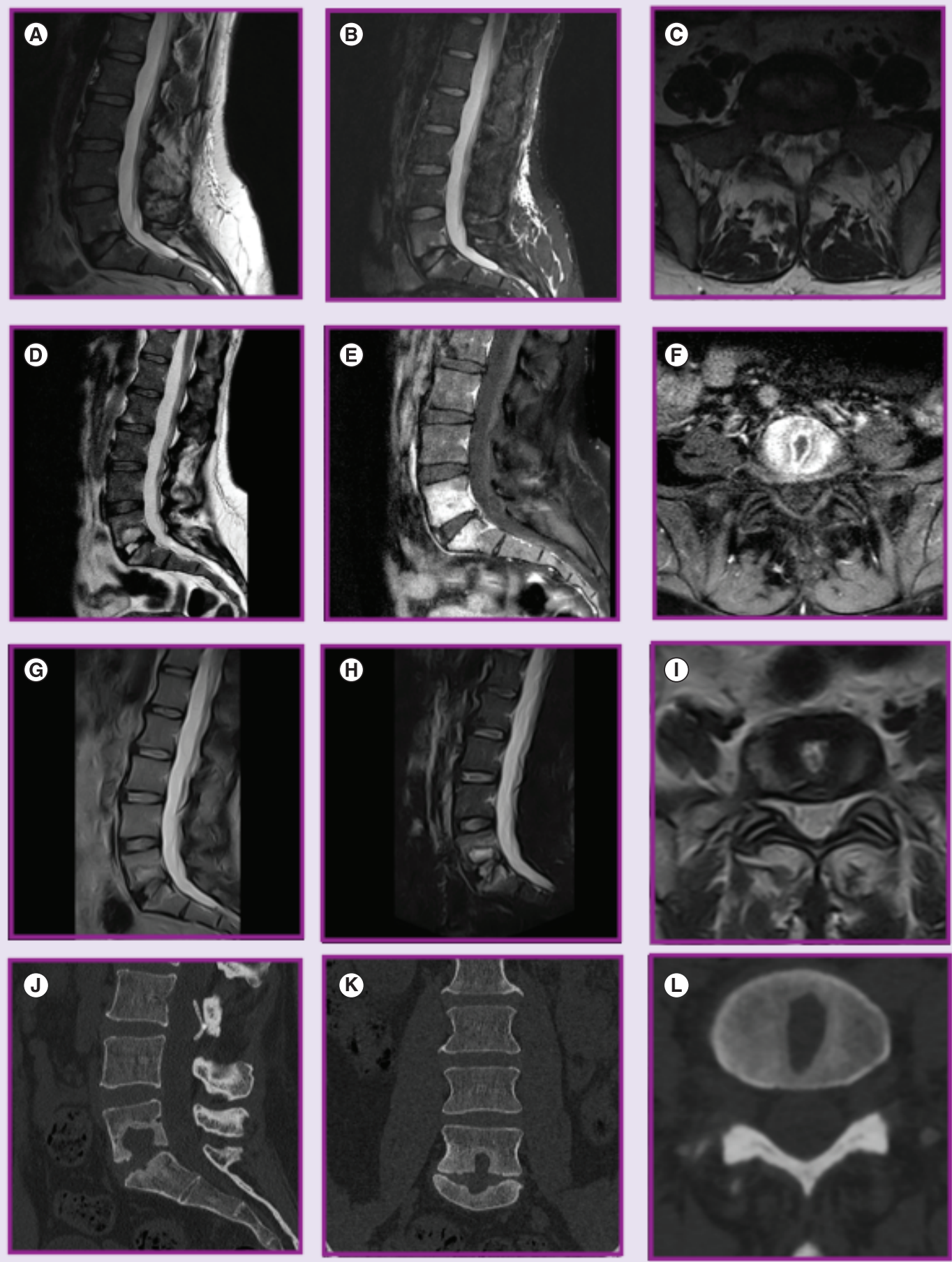

Figure 2. Postprocedure lumbar spine imaging. MRI lumbar spine without contrast 10 weeks postprocedure (A-C) with sagittal T2, sagittal STIR and axial T2 images; MRI lumbar spine with and without contrast 14 weeks postprocedure (D-F) with sagittal T2, sagittal T2 postcontrast and axial T2 postcontrast images; MRI lumbar spine without contrast 8 months postprocedure (G-I) with sagittal T2, sagittal STIR and axial T2 images; CT scan lumbar spine 8 months postprocedure (J-L) with sagittal, coronal and axial images. 
contrast medium. With the use of orthobiologics and IBT, there is evidence that antibiotics and contrast media alter the viability of cells and biologic activity $[20,40,41]$ and therefore are judiciously utilized based on the risk and benefits involved. This reduction or lack of antibiotic use potentially increases the risk of infection compared to routine discography. Better understanding of the role and route of antibiotics in preventing C. acnes spondylodiscitis and its effect on treatment outcomes is needed.

At last, the role of each component of the intradiscal biologic injectate in healing needs better understanding. PRP has antimicrobial properties useful in clinical applications $[19,22]$ that is not fully understood - attributed to platelets, alpha granule contents such as thrombocidins, leukocytes [42] and plasma peptides [21]. There have been several studies comparing the effects of leukocyte concentration on antimicrobial properties of autologous PRP with the overall conclusion that the leukocyte concentration was not correlated with any change in antimicrobial effects, suggesting it may be the plasma or platelet components rather than the leukocytes [24]. However, other research concludes that only high concentrations of leukocytes inhibited Staphylococcus epidermidis [43]. When treating the intervertebral disc, Wang [44] concludes that leukocytes in PRP activate inflammatory and catabolic effects on nucleus pulposus-derived stem cells and therefore recommend leukocyte-poor PRP for intervertebral disc disease. This inflammatory response may mimic non-pyogenic discitis which has been anecdotally described following IBT. By improving our understanding of the injectate mechanisms of action, we will be able to devise better treatment options.

\section{Conclusion}

The current case highlights the need for better understanding of the infection risks associated with IBT as the utilization of these treatments increase. Additional studies are needed to learn whether or not different types of PRP preparations provide greater protection against $C$. acne spondylodiscitis [37]. In addition, further studies are needed to see whether or not the addition of intradiscal and/or intravenous antibiotics at the time of IBT will adversely affect clinical outcomes.

\section{Summary points}

- The native regenerative potential of the intervertebral disc declines with aging and may be augmented by autologous orthobiologic treatments.

- Intradiscal biologic therapies (IBT) including intradiscal platelet-rich plasma (PRP) injections may result in spondylodiscitis.

- Cutibacterium acnes (formerly Propionibacterium acnes) is an aerotolerant anaerobic Gram-positive rod-shaped bacterium that can be prevalent in intervertebral discs of asymptomatic patients, but is also a well-established pathogenic bacterium resulting in spondylodiscitis.

- In this case, C. acnes was isolated on biopsy with heavy growth and associated pus consistent with pyogenic spondylodiscitis. This species and its subtypes are an under-recognized pathogen in spine infections, complicated by its more indolent clinical presentation, minimal laboratory signs and challenges in culture due to questions of its role as a pathogen or contaminate.

- While rare, this case highlights the potential morbidity associated with IBT infections.

- Prevention of IBT-associated spondylodiscitis requires: vigilance in sterile technique, including biologic injectate preparation; risk-stratification of patients who might be at higher risk for infection; and improved protocols based on better understanding of the mechanisms involved in the pleiotropic effects of PRP and its interactions with the in vivo intervertebral disc environment.

- PRP preparation protocols and its antimicrobial properties need further study in the context $C$. acnes which can resist phagocytosis and persist in macrophages, mediate inflammatory cascades and exhibit cell-aderant properties that may play a role in biofilm formation.

- Further research is needed to better understand the ideal type, dose and route of antibiotics with IBT. The potential risk of decreasing cellular viability and growth factor activity must be weighed against the benefit of reducing risk of spondylodiscitis. Traditional antibiotic treatment protocols for discography and nonbiologic treatments provide some guidance but need further study in the context of regenerative medicine.

- The early inflammatory response anecdotally observed in IBT may mimic nonpyogenic or pyogenic discitis. This phenomenon may ultimately be a key component of successful IBT or may be unnecessary and possibly minimized through the alteration of the biologic preparation protocol, adjusting for cell types and cytokine make-up.

- This case highlights the need for better understanding of the infection risks associated with IBT as the utilization of these treatments increase. Additional studies are needed to see whether or not different types of PRP preparations and different antibiotic protocols provide greater protection against C. Acnes spondylodiscitis. 


\section{Financial \& competing interests disclosure}

The authors have no relevant affiliations or financial involvement with any organization or entity with a financial interest in or financial conflict with the subject matter or materials discussed in the manuscript. This includes employment, consultancies, honoraria, stock ownership or options, expert testimony, grants or patents received or pending, or royalties.

No writing assistance was utilized in the production of this manuscript.

\section{Ethical conduct of research}

The authors state that they have obtained verbal and written informed consent from the patient/patients for the inclusion of their medical and treatment history within this case report.

\section{Open access}

This work is licensed under the Attribution-NonCommercial-NoDerivatives 4.0 Unported License. To view a copy of this license, visit http://creativecommons.org/licenses/by-nc-nd/4.0/

\section{References}

1. Meucci RD, Fassa AG, Faria NM. Prevalence of chronic low back pain: systematic review. Rev. Saude Publica 49, doi:10.1590/S0034-8910.2015049005874 (2015).

2. Husky MM, Ferdous Farin F, Compagnone P, Fermanian C, Kovess-Masfety V. Chronic back pain and its association with quality of life in a large French population survey. Health. Qual. Life. Outcomes 16(1), 4 (2018).

3. Hurwitz EL, Randhawa K, Yu H, Cote P, Haldeman S. The Global Spine Care Initiative: a summary of the global burden of low back and neck pain studies. Eur. Spine J. 27(Suppl. 6), 796-801 (2018).

4. Katz JN. Lumbar disc disorders and low-back pain: socioeconomic factors and consequences. J. Bone Joint Surg. Am. 88(Suppl. 2), 21-24 (2006).

5. Roberts S, Evans H, Trivedi J, Menage J. Histology and pathology of the human intervertebral disc. J. Bone Joint Surg. Am. 88(Suppl. 2), $10-14$ (2006).

6. Salzmann SN, Plais N, Shue J, Girardi FP. Lumbar disc replacement surgery-successes and obstacles to widespread adoption. Curr. Rev Musculoskelet. Med. 10(2), 153-159 (2017).

7. Mannion AF, Brox JI, Fairbank JC. Comparison of spinal fusion and nonoperative treatment in patients with chronic low back pain: long-term follow-up of three randomized controlled trials. Spine J. 13(11), 1438-1448 (2013).

8. Alini M, Roughley PJ, Antoniou J, Stoll T, Aebi M. A biological approach to treating disc degeneration: not for today, but maybe for tomorrow. Eur. Spine J. 11(Suppl. 2), 215 (2002).

9. Bendtsen M, Bunger C, Colombier P et al. Biological challenges for regeneration of the degenerated disc using cellular therapies. Acta Orthop. 87(Suppl. 363), 39-46 (2016).

10. Wu H, Shang Y, Yu J et al. Regenerative potential of human nucleus pulposus resident stem/progenitor cells declines with ageing and intervertebral disc degeneration. Int. J. Mol. Med. 42(4), 2193-2202 (2018).

11. Vasiliadis ES, Pneumaticos SG, Evangelopoulos DS, Papavassiliou AG. Biologic treatment of mild and moderate intervertebral disc degeneration. Mol. Med. 20(1), 400-409 (2014).

12. Tong W, Lu Z, Qin L et al. Cell therapy for the degenerating intervertebral disc. Transl. Res. 181, 49-58 (2017).

13. Rosenberg GJ, Yee AJM, Erwin WM. Bedside to bench and back to bedside: translational implications of targeted intervertebral disc therapeutics. J. Orthop. Translat. 10, 18-27 (2017).

14. Akeda $\mathrm{K}$, Ohishi $\mathrm{K}$, Masuda $\mathrm{K}$ et al. Intradiscal injection of autologous platelet-rich plasma releasate to treat discogenic low back pain: a preliminary clinical trial. Asian Spine J. 11(3), 380-389 (2017).

15. Tuakli-Wosornu YA, Terry A, Boachie-Adjei K et al. Lumbar intradiskal platelet-rich plasma (PRP) injections: a prospective, double-blind, randomized controlled study. PM R 8(1), 10 (2016).

16. Levi D, Horn S, Tyszko S, Levin J, Hecht-Leavitt C, Walko E. Intradiscal platelet-rich plasma injection for chronic discogenic low back pain: preliminary results from a prospective trial. Pain Med. 17(6), 1010-1022 (2016).

17. Monfett M, Harrison J, Boachie-Adjei K, Lutz G. Intradiscal platelet-rich plasma (PRP) injections for discogenic low back pain: an update. Int. Orthop. 40(6), 1321-1328 (2016).

18. Mohammed S, Yu J. Platelet-rich plasma injections: an emerging therapy for chronic discogenic low back pain. J. Spine Surg. 4(1), $115-122$ (2018).

19. Badade PS, Mahale SA, Panjwani AA, Vaidya PD, Warang AD. Antimicrobial effect of platelet-rich plasma and platelet-rich fibrin. Indian J. Dent. Res. 27(3), 300-304 (2016).

20. Intravia J, Allen DA, Durant TJ et al. In vitro evaluation of the anti-bacterial effect of two preparations of platelet rich plasma compared with cefazolin and whole blood. Muscles Ligaments Tendons J. 4(1), 79-84 (2014). 
21. Anitua E, Andia I, Ardanza B, Nurden P, Nurden AT. Autologous platelets as a source of proteins for healing and tissue regeneration. Thromb. Haemost. 91(1), 4-15 (2004).

22. Yang LC, Hu SW, Yan M, Yang JJ, Tsou SH, Lin YY. Antimicrobial activity of platelet-rich plasma and other plasma preparations against periodontal pathogens. J. Periodontol. 86(2), 310-318 (2015).

23. Li H, Li B. PRP as a new approach to prevent infection: preparation and in vitro antimicrobial properties of PRP. J. Vis. Exp. (2013). https://www.ncbi.nlm.nih.gov/pmc/articles/PMC3653398/

24. D'asta F, Halstead F, Harrison P, Zecchi Orlandini S, Moiemen N, Lord J. The contribution of leucocytes to the antimicrobial activity of platelet-rich plasma preparations: a systematic review. Platelets 29(1), 9-20 (2018).

25. Peh W. Provocative discography: current status. Biomed. Imaging Interv. J. 1(1), e2 (2005).

26. Schellhas KP, Pollei SR, Gundry CR, Heithoff KB. Lumbar disc high-intensity zone. Correlation of magnetic resonance imaging and discography. Spine 21(1), 79-86 (1996).

27. Pobiel RS, Schellhas KP, Pollei SR, Johnson BA, Golden MJ, Eklund JA. Diskography: infectious complications from a series of 12,634 cases. Am. J. Neuroradiol. 27(9), 1930-1932 (2006).

28. McDonald M, Spelman D, Baron E. Vertebral osteomyelitis and discitis in adults. UptoDate 11-21 (2019). https://www.uptodate.com/contents/vertebral-osteomyelitis-and-discitis-in-adults

29. Leheste JR, Ruvolo KE, Chrostowski JE et al. P. acnes-driven disease pathology: current knowledge and future directions. Front. Cell. Infect. Microbiol. 7, 81 (2017).

30. Stirling A, Worthington T, Rafiq M, Lambert PA, Elliott TS. Association between sciatica and Propionibacterium acnes. Lancet 357(9273), 2024-2025 (2001).

31. Aubin GG, Portillo ME, Trampuz A, Corvec S. Propionibacterium acnes, an emerging pathogen: from acne to implant-infections, from phylotype to resistance. Med. Mal. Infect. 44(6), 241-250 (2014).

32. Saper D, Capiro N, Ma R, Li X. Management of Propionibacterium acnes infection after shoulder surgery. Curr. Rev. Musculoskelet. Med. 8(1), 67-74 (2015).

33. Capoor MN, Ruzicka F, Schmitz JE et al. Propionibacterium acnes biofilm is present in intervertebral discs of patients undergoing microdiscectomy. PLoS ONE 12(4), e0174518 (2017).

34. Chen Z, Cao P, Zhou Z, Yuan Y, Jiao Y, Zheng Y. Overview: the role of Propionibacterium acnes in nonpyogenic intervertebral discs. Int. Orthop. 40(6), 1291-1298 (2016).

35. Harris AE, Hennicke C, Byers K, Welch WC. Postoperative discitis due to Propionibacterium acnes: a case report and review of the literature. Surg. Neurol. 63(6), 541; discussion 541 (2005).

36. Uckay I, Dinh A, Vauthey L et al. Spondylodiscitis due to Propionibacterium acnes: report of twenty-nine cases and a review of the literature. Clin. Microbiol. Infect. 16(4), 353-358 (2010).

37. Webster GF, Leyden JJ, Musson RA, Douglas SD. Susceptibility of Propionibacterium acnes to killing and degradation by human neutrophils and monocytes in vitro. Infect. Immun. 49(1), 116-121 (1985).

38. Klessig HT, Showsh SA, Sekorski A. The use of intradiscal antibiotics for discography: an in vitro study of gentamicin, cefazolin, and clindamycin. Spine 28(15), 1735-1738 (2003).

39. Kim D, Wadley R. Variability in techniques and patient safety protocols in discography: a national multispecialty survey of International Spine Intervention Society members. J. Spinal. Disord. Tech. 23(6), 431-438 (2010).

40. Parsha K, Mir O, Satani $\mathrm{N}$ et al. Mesenchymal stromal cell secretomes are modulated by suspension time, delivery vehicle, passage through catheter, and exposure to adjuvants. Cytotherapy 19(1), 36-46 (2017).

41. Wu T, Nie H, Dietz AB et al. Cytotoxic effects of nonionic iodinated contrast agent on human adipose-derived mesenchymal stem cells. PM R (2018). https://onlinelibrary.wiley.com/doi/10.1016/j.pmrj.2018.05.022

42. Bielecki TM, Gazdzik TS, Arendt J, Szczepanski T, Krol W, Wielkoszynski T. Antibacterial effect of autologous platelet gel enriched with growth factors and other active substances: an in vitro study. J. Bone Joint Surg. Br. 89(3), 417-420 (2007).

43. Anitua E, Alonso R, Girbau C, Aguirre JJ, Muruzabal F, Orive G. Antibacterial effect of plasma rich in growth factors (PRGF(R)-Endoret(R)) against Staphylococcus aureus and Staphylococcus epidermidis strains. Clin. Exp. Dermatol. 37(6), 652-657 (2012).

44. Wang SZ, Fan WM, Jia J, Ma LY, Yu JB, Wang C. Is exclusion of leukocytes from platelet-rich plasma (PRP) a better choice for early intervertebral disc regeneration? Stem Cell. Res. Ther. 9(1), 7 (2018). 